Vietnam Journal of Mechanics, VAST, Vol.43, No. 1 (2021), pp. $91-104$

DOI: https://doi.org/10.15625/0866-7136/15774

\title{
THEORETICAL MODEL OF GUIDED WAVES IN A BONE-MIMICKING PLATE COUPLED WITH SOFT-TISSUE LAYERS
}

\author{
Hoai Nguyen ${ }^{1}$, Ductho Le ${ }^{2}$, Emmanuel Plan ${ }^{3,4}$, Son Tung Dang ${ }^{5}$, Haidang Phan ${ }^{3,6}$ \\ ${ }^{1}$ Institute of Physics, Vietnam Academy of Science and Technology, Hanoi, Vietnam \\ ${ }^{2}$ Faculty of Mathematics, Mechanics and Informatics, VNU University of Science, Vietnam \\ ${ }^{3}$ Institute of Theoretical and Applied Research, Duy Tan University, Hanoi, Vietnam \\ ${ }^{4}$ Faculty of Natural Science, Duy Tan University, Da Nang, Vietnam \\ ${ }^{5}$ Sintef industry, S. P. Andersens veg 15B, 7031 Trondheim, Norway \\ ${ }^{6}$ Faculty of Civil Engineering, Duy Tan University, Da Nang, Vietnam \\ *E-mail: phanhaidang2@duytan.edu.vn
}

Received: 21 December 2020 / Published online: 21 February 2021

\begin{abstract}
Quantitative ultrasound has shown a significant promise in the assessment of bone characteristics in the recent reports. However, our understanding of wave interaction with bone tissues is still far from complete since the propagation of ultrasonic waves in bones is a very challenging topic due to their multilayer nature. The aim of the current study is to develop a theoretical model for guided waves in a bone-mimicking plate coupled with two soft-tissue layers. Here, the bone plate is modeled as an isotropic solid layer while the soft tissues are modeled as fluid layers. Based on the boundary conditions set for the three-layered structure, a characteristic equation is obtained which results in dispersion curves of the phase and group velocities. New expressions for free guided waves propagating in the trilayered plate are introduced. The amplitudes of wave modes generated by time-harmonic loads applied in the plate are theoretically computed by reciprocity consideration. As an example of calculation, the normalized amplitudes of the lowest wave modes are presented. The obtained results and equations discussed in this study could be, in general, useful for further applications in the area of bone quantitative ultrasound.
\end{abstract}

Keywords: guided waves; bone plate; trilayered structures; reciprocity; quantitative ultrasound.

\section{INTRODUCTION}

Guided wave propagation in layered structures plays an important role in the study of quantitative ultrasound (QUS), a method of great potential in the assessment of bone characteristics. Bone QUS takes advantage of mechanical waves that are more sensitive 
than conventional X-ray method to the determinants of bone strength [1]. Unlike Xrays, QUS is safe for newborn babies and pregnant women because it is a non-ionizing method. Moreover, QUS approach can provide information about the elastic properties and defects of bones [2]. Numerous studies have been considered to understand how ultrasound interacts with the bone structure, see, for example, $[3,4]$. Lowet and Van der Perre [5] studied simulation of ultrasound wave propagation and the method to measure velocity in long bones. Numerical simulations of wave propagation and experiment measurement were used to gain insights into the expected behavior of guided waves in bones [6]. The velocity dispersion and attenuation in a tri-layered system, which consists of a transversely-isotropic cortical bone plate sandwiched between the soft-tissue and marrow layers, were computed using a semi-analytical finite element [7]. However, wave propagation and scattering in bones is a very challenging topic due to the bones' multi-layer, anisotropic, and viscoelastic nature. The understanding of wave interaction with bones is, therefore, still quite limited and definitely needs to be expanded $[1,3]$.

Wave propagation in multi-layered structures is unquestionably one of the most fundamental problems of elastodynamics. Study of free guided waves in layered plates can be found in textbooks $[8,9]$ and research papers [10,11]. The dispersion equation of guided waves in fluid-solid bilayered plate is discussed in [12]. The phenomenon of osculation where two dispersion curves come near to each other was observed and carefully studied by [13]. Wave motion generated by a loading is in general solved by the use of integral transform technique [8,14-16] and by the reciprocity approach [17-27]. The integral transform approach is usually used for simple half-space problems. However, it becomes more difficult for anisotropic solids, and impossible for inhomogeneous solids. The reciprocity approach is therefore suitable for guided wave motions in layered structures and composites.

In this work, we present a model for ultrasonic guided waves in a trilayered system consisting of a bone-mimicking plate coupled with a soft-tissue layer and a marrow layer. In order to simplify the computation procedure, we consider the bone layer as an isotropic solid while the soft-tissue and the marrow layer as pure fluids. For modeling purpose, we also ignore the effect of viscosity. For this trilayered structure, boundary conditions are suitably applied at the free surfaces of fluids and the interfaces between the solid and the fluids to derive dispersion equations. Based on these equations, dispersion curves are obtained by numerical computation. Explicit expressions of displacements and stresses in the trilayered structures are then introduced. The amplitudes of guided waves generated by a time-harmonic load applied in the solid plate are theoretically computed by using reciprocity in elastodynamics. As an example, the normalized amplitudes of the lowest wave modes are computed.

\section{EXPRESSIONS FOR GUIDED WAVES IN A FLUID-SOLID-FLUID PLATE}

Let us consider a solid layer $\Omega$ representing the bone plate bonded in the middle of two fluid layers $\hat{\Omega}$ and $\tilde{\Omega}$ which presents the upper and lower soft tissues, respectively. This forms a fluid-solid-fluid layered plate in Cartesian coordinate system $(x, y, z)$, as shown in Fig. 1. Propagation of free guided waves in the trilayered structure is of interest 
in this section. The dispersion equation is derived resulting in the dispersion curves. In order to perform direct application of reciprocity theorem in the next section, new expressions of displacement and stress components are also introduced.

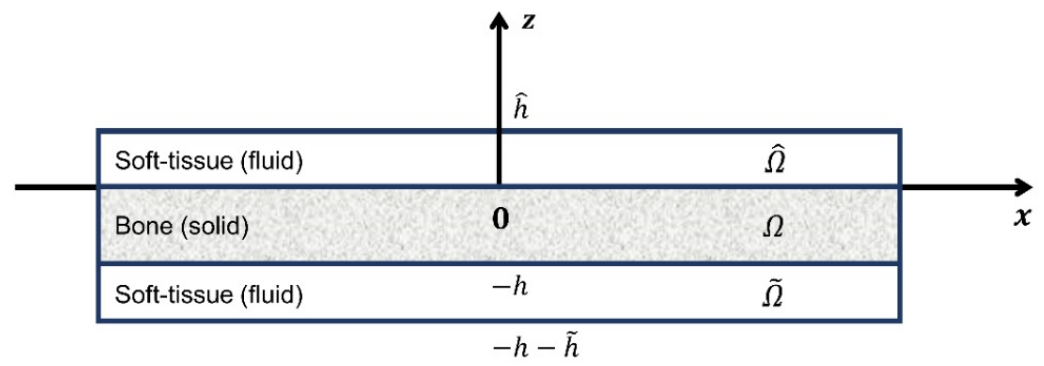

Fig. 1. Coordinate system for trilayered plate

Besides the amplitudes, guided waves in the fluid-solid-fluid plate are characterized by an angular frequency $\omega$ and a wavenumber $k$, where $k=\omega / c, c$ being the phase velocity, as well as Lame constants $\hat{\lambda}, \hat{\mu}, \lambda, \mu$ and $\tilde{\lambda}, \tilde{\mu}$, and densities $\hat{\rho}, \rho$ and $\tilde{\rho}$ of the upper fluid, the solid and the lower fluid, respectively. Note that the non-viscous fluids considered here do not sustain shear stresses and their equations of motion can be used as of a solid by assuming Lame constants $\hat{\mu}=0, \tilde{\mu}=0$. The upper fluid layer's thickness is indicated by $\hat{h}$, the one of the solid is indicated by $h$ and the lower fluid layer's thickness is denoted by $\tilde{h}$. Wave solutions are expressed based on the partial wave theory discussed in detail, for example, in [9]. Since there are two partial waves in the upper fluid layer, the displacements may be written as

$$
\begin{aligned}
& \hat{u}_{x}=\left(\hat{A}_{1} e^{i k \hat{\alpha} z}+\hat{A}_{2} e^{-i k \hat{\alpha} z}\right) e^{i k(x-c t)}, \\
& \hat{u}_{z}=\hat{\alpha}\left(\hat{A}_{1} e^{i k \hat{\alpha} z}-\hat{A}_{2} e^{-i k \hat{\alpha} z}\right) e^{i k(x-c t)} .
\end{aligned}
$$

For the solid layer, the number of partial waves is four. Thus, the displacement components are of the form

$$
\begin{aligned}
& u_{x}=\left(A_{1} e^{i k \alpha_{1} z}+A_{2} e^{i k \alpha_{2} z}+A_{3} e^{-i k \alpha_{1} z}+A_{4} e^{-i k \alpha_{2} z}\right) e^{i k(x-c t)} \\
& u_{z}=\left(\frac{-1}{\alpha_{1}} A_{1} e^{i k \alpha_{1} z}+\alpha_{2} A_{2} e^{i k \alpha_{2} z}+\frac{1}{\alpha_{1}} A_{3} e^{-i k \alpha_{1} z}-\alpha_{2} A_{4} e^{-i k \alpha_{2} z}\right) e^{i k(x-c t)} .
\end{aligned}
$$

Since there are two partial waves in the lower fluid layer, the displacements may be written as

$$
\begin{aligned}
& \tilde{u}_{x}=\left(\tilde{A}_{1} e^{i k \tilde{\alpha} z}+\tilde{A}_{2} e^{-i k \tilde{\alpha} z}\right) e^{i k(x-c t)}, \\
& \tilde{u}_{z}=\tilde{\alpha}\left(\tilde{A}_{1} e^{i k \tilde{\alpha} z}-\tilde{A}_{2} e^{-i k \tilde{\alpha} z}\right) e^{i k(x-c t)} .
\end{aligned}
$$


Here, $\hat{A}_{j}(j=1,2), A_{j}(j=1,2,3,4)$ and $\tilde{A}_{j}(j=1,2)$ are constants to be determined. The dimensionless quantities are defined as follow

$$
\hat{\alpha}=\sqrt{\left(-1+c^{2} / \hat{c}_{L}^{2}\right)},
$$

where

$$
\hat{c}_{L}=\sqrt{\hat{\lambda} / \hat{\rho}},
$$

are the longitudinal wave velocity of the fluid layer $\hat{\Omega}$. Similarly,

$$
\alpha_{1}=\sqrt{-1+c^{2} / c_{T}^{2}}, \quad \alpha_{2}=\sqrt{-1+c^{2} / c_{L}^{2}},
$$

where

$$
c_{T}=\sqrt{\mu / \rho}, \quad c_{L}=\sqrt{(\lambda+2 \mu) / \rho},
$$

are the transverse and longitudinal wave velocities, respectively, of the solid layer $\Omega$. For the lower fluid layer

$$
\tilde{\alpha}=\sqrt{\left(-1+c^{2} / \tilde{c}_{L}^{2}\right)},
$$

where

$$
\tilde{c}_{L}=\sqrt{\tilde{\lambda} / \tilde{\rho}}
$$

Note that the corresponding stress components $\hat{\tau}_{x x}, \hat{\tau}_{z z}$ of the layer $\hat{\Omega}, \tau_{x x}, \tau_{x z}, \tau_{z z}$ of the layer $\Omega$ and $\tilde{\tau}_{x x}, \tilde{\tau}_{z z}$ of the layer $\tilde{\Omega}$ can be easily calculated by the use of Hooke's law. The boundary conditions are written as

$$
\begin{array}{ll}
\hat{\tau}_{z z}=0 & (z=\hat{h}) \\
u_{z}=\hat{u}_{z}, \tau_{x z}=0, \tau_{z z}=\hat{\tau}_{z z} & (z=0) \\
u_{z}=\tilde{u}_{z}, \tau_{x z}=0, \tau_{z z}=\tilde{\tau}_{z z} & (z=-h) \\
\tilde{\tau}_{z z}=0 & (z=-(h+\tilde{h}))
\end{array}
$$

Eq. (13), after some manipulation, results in

$$
\mathbf{D A}=\mathbf{0},
$$

where

$$
\mathbf{A}=\left[\begin{array}{llllllll}
\hat{A}_{1} & \hat{A}_{2} & A_{1} & A_{2} & A_{3} & A_{4} & \tilde{A}_{1} & \tilde{A}_{2}
\end{array}\right]^{T},
$$

and $\mathbf{D}$ is the eight-by-eight matrix whose expression is given in Appendix A, see Eq. (A.1).

In order to have nontrivial solutions, the determinant of the eight-by-eight matrix in Eq. (14) must be zero. This is referred to as the characteristic equation of guided waves in the fluid-solid-fluid plate with phase velocity as an unknown parameter when properties of the three layers and frequency are given. Since the determinant appears with a frequency term via the wavenumber $k$, the phase velocity is dependent of frequency. Therefore, guided waves in the trilayered are dispersive. Note that the characteristic equation in general has infinite number of solutions, each of which corresponds to one 
wave mode. In the examples shown in Fig. 2, the relevant material properties are tabulated in Tab. 1. The computation of the dispersion curves for a water-aluminum-water trilayered structure in Fig. 2 coincides with the one obtained by Nguyen et al. [7].

Table 1. Material properties of aluminum and water

\begin{tabular}{cccc}
\hline Material & $\rho\left(\mathrm{kg} / \mathrm{m}^{3}\right)$ & $\lambda(\mathrm{GPa})$ & $\mu(\mathrm{GPa})$ \\
\hline Water & 1000 & 2.25 & 0 \\
Aluminum & 2700 & 55.25 & 25.94 \\
\hline
\end{tabular}

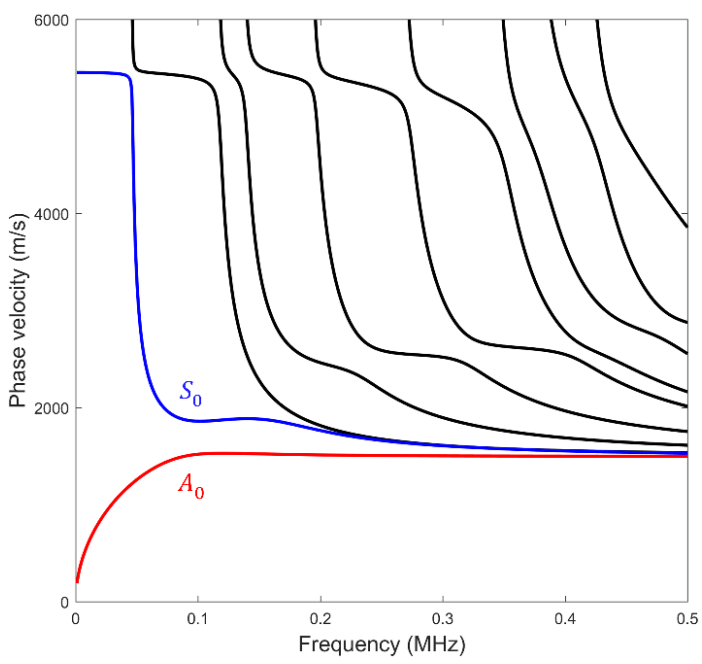

(a)

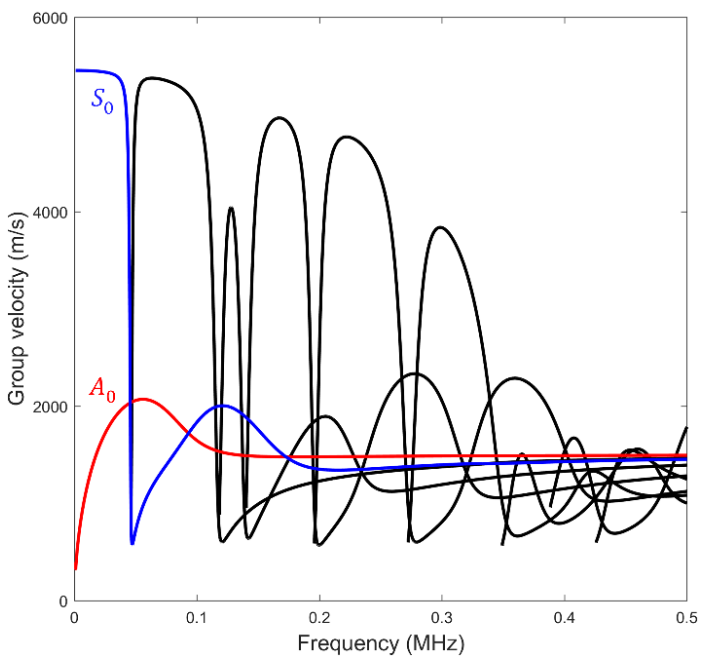

(b)

Fig. 2. Dispersion curves of phase and group velocities: Lowest symmetric mode $S_{0}$ (blue),

Lowest antisymmetric mode $A_{0}$ (red) and Higher modes (black)

In Eq. (14), there are actually seven independent equations with eight unknowns $\hat{A}_{1}, \hat{A}_{2}, A_{1}, A_{2}, A_{3}, A_{4}$, and $\tilde{A}_{1}, \tilde{A}_{2}$. We find solution of Eq. (14) in the form of$$
\hat{A}_{1}=A \hat{d}_{1}, \hat{A}_{2}=A \hat{d}_{2}, A_{1}=A d_{1}, A_{2}=A d_{2}, A_{3}=A d_{3}, A_{4}=A d_{4}, \tilde{A}_{1}=A \tilde{d}_{1}, \tilde{A}_{2}=A \tilde{d}_{2}
$$

where $A$ is only unknown and $\hat{d}_{1}, \hat{d}_{2}, d_{1}, d_{2}, d_{3}, d_{4}, \tilde{d}_{1}, \tilde{d}_{2}$ are dimensionless quantities depending on material properties and thicknesses of the fluids and the solid, and the wavenumber $k$. The expressions of $\hat{d}_{1}, \hat{d}_{2}, d_{1}, d_{2}, d_{3}, d_{4}, \tilde{d}_{1}, \tilde{d}_{2}$ are given in Appendix A, see Eq. (A.2). With the introduction of relative amplitude $A$, we may now rewrite the displacement and stress fields as follows:

- For the upper fluid layer

$$
\begin{aligned}
\hat{u}_{x} & =A \hat{U}_{x}(z) e^{i k(x-c t),} \\
\hat{u}_{z} & =A \hat{U}_{z}(z) e^{i k(x-c t),} \\
\hat{\tau}_{x x} & =i k \lambda A \hat{T}_{x x}(z) e^{i k(x-c t),}
\end{aligned}
$$


where

$$
\begin{aligned}
\hat{U}_{x}(z) & =\hat{d}_{1} e^{i k \hat{\alpha} z}+\hat{d}_{2} e^{-i k \hat{\alpha} z}, \\
\hat{U}_{z}(z) & =\alpha\left(\hat{d}_{1} e^{i k \hat{\alpha} z}-\hat{d}_{2} e^{-i k \hat{\alpha} z}\right), \\
\hat{T}_{x x}(z) & =\left(1+\hat{\alpha}^{2}\right)\left(\hat{d}_{1} e^{i k \hat{\alpha} z}+\hat{d}_{2} e^{-i k \hat{\alpha} z}\right) .
\end{aligned}
$$

- For the solid layer

$$
\begin{aligned}
u_{x} & =A U_{x}(z) e^{i k(x-c t),} \\
u_{z} & =A U_{z}(z) e^{i k(x-c t),} \\
\tau_{x x} & =i k \mu A T_{x x}(z) e^{i k(x-c t),}, \\
\tau_{x z} & =i k \mu A T_{x z}(z) e^{i k(x-c t),}
\end{aligned}
$$

where

$$
\begin{aligned}
U_{x}(z) & =d_{1} e^{i k \alpha_{1} z}+d_{2} e^{i k \alpha_{2} z}+d_{3} e^{-i k \alpha_{1} z}+d_{4} e^{-i k \alpha_{2} z} \\
U_{z}(z) & =\frac{-1}{\alpha_{1}} d_{1} e^{i k \alpha_{1} z}+\alpha_{2} d_{2} e^{i k \alpha_{2} z}+\frac{1}{\alpha_{1}} d_{3} e^{-i k \alpha_{1} z}-\alpha_{2} d_{4} e^{-i k \alpha_{2} z}, \\
T_{x x}(z) & =2 d_{1} e^{i k \alpha_{1} z}+\left(\alpha_{1}^{2}-2 \alpha_{2}^{2}+1\right) d_{2} e^{i k \alpha_{2} z}+2 d_{3} e^{-i k \alpha_{1} z}+\left(\alpha_{1}^{2}-2 \alpha_{2}^{2}+1\right) d_{4} e^{-i k \alpha_{2} z}, \\
T_{x z}(z) & =\left(\alpha_{1}-\frac{1}{\alpha_{1}}\right) d_{1} e^{i k \alpha_{1} z}+2 \alpha_{2} d_{2} e^{i k \alpha_{2} z}-\left(\alpha_{1}-\frac{1}{\alpha_{1}}\right) d_{3} e^{-i k \alpha_{1} z}-2 \alpha_{2} d_{4} e^{-i k \alpha_{2} z},
\end{aligned}
$$

- For the lower fluid layer

$$
\begin{aligned}
\tilde{u}_{x} & =A \tilde{U}_{x}(z) e^{i k(x-c t),} \\
\tilde{u}_{z} & =A \tilde{U}_{z}(z) e^{i k(x-c t),} \\
\tilde{\tau}_{x x} & =i k \lambda A \tilde{T}_{x x}(z) e^{i k(x-c t),}
\end{aligned}
$$

where

$$
\begin{aligned}
\tilde{U}_{x}(z) & =\tilde{d}_{1} e^{i k \tilde{\alpha} z}+\tilde{d}_{2} e^{-i k \tilde{\alpha} z}, \\
\tilde{U}_{z}(z) & =\alpha\left(\tilde{d}_{1} e^{i k \tilde{\alpha} z}-\tilde{d}_{2} e^{-i k \tilde{\alpha} z}\right), \\
\tilde{T}_{x x}(z) & =\left(1+\tilde{\alpha}^{2}\right)\left(\tilde{d}_{1} e^{i k \tilde{\alpha} z}+\tilde{d}_{2} e^{-i k \tilde{\alpha} z}\right) .
\end{aligned}
$$

Instead of depending on eight unknowns as given in Eqs. (1)-(6), the guided wave fields now depend only on the amplitude $A$. Although guided waves in a plate are usually separated into symmetric and antisymmetric modes, the displacement and stress fields given by Eqs. (17)-(36) already include both symmetric and antisymmetric modes. The main reason to introduce these expressions is to conveniently apply the reciprocity theorems and they are very important to the problem of guided wave motions subjected to time-harmonic loads in trilayered plates discussed in the next section. 


\section{COMPUTATION OF GUIDED WAVES DUE TO TIME-HARMONIC LOADING}

Considered in this section is the computation of guided wave motions in the trilayered structure (Fig. 1) subjected to a time-harmonic line load. Suppose that we have a vertical load $f_{z}^{A}$ at $\left(x_{0}, z_{0}\right)$ where $x_{0}, z_{0}$ are the $x$-coordinate and the $z$-coordinate, respectively, of the point of application. The load demonstrated in Fig. 3 may be written as

$$
f_{z}^{A}=P \delta\left(z-z_{0}\right) \delta\left(x-x_{0}\right) e^{-i k c t} .
$$

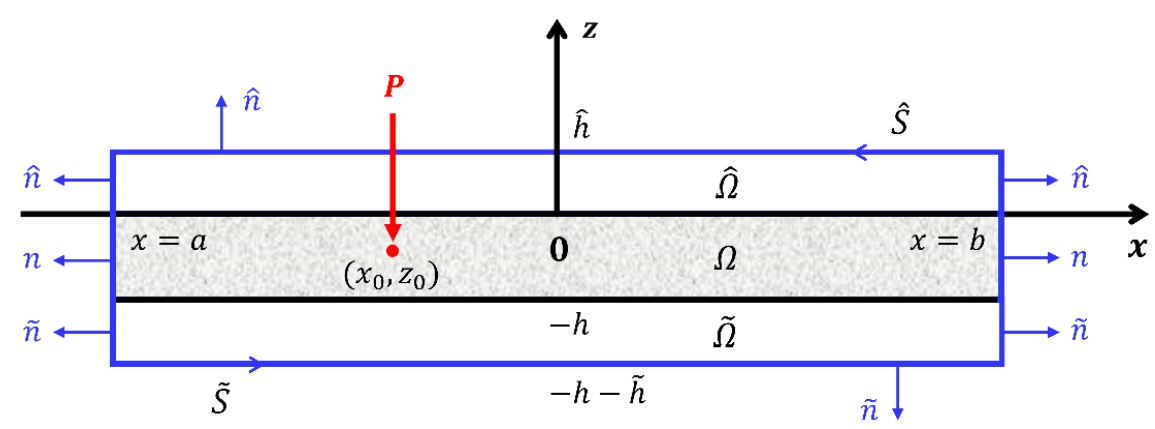

Fig. 3. Trilayered structure due to time-harmonic loading

This load will generate various guided wave modes along the fluid-solid-fluid plate in both the positive $x$-direction and the negative $x$-direction with unknown scattered amplitudes $A_{m}^{P^{+}}$and $A_{m}^{P_{-}}(m=0,1, \ldots, \infty)$, respectively. State $A$ is called the actual state whose amplitudes will be determined using the reciprocity relation for a three-material body. Since many wave modes are generated in the trilayered structure, the far-field displacements of each layer of state $A$ in the positive $x$-direction may be written in the form of

$$
\begin{aligned}
& \hat{u}_{x}=\sum_{m=0}^{\infty} \hat{u}_{x}^{m}=\sum_{m=0}^{\infty} A_{m}^{P+} \hat{U}_{x}^{m}(z) e^{i k_{m}\left(x-c_{m} t\right)}, \\
& \hat{u}_{z}=\sum_{m=0}^{\infty} \hat{u}_{z}^{m}=-i \sum_{m=0}^{\infty} A_{m}^{P+} \hat{U}_{z}^{m}(z) e^{i k_{m}\left(x-c_{m} t\right)}, \\
& u_{x}=\sum_{m=0}^{\infty} u_{x}^{m}=\sum_{m=0}^{\infty} A_{m}^{P+} U_{x}^{m}(z) e^{i k_{m}\left(x-c_{m} t\right)}, \\
& u_{z}=\sum_{m=0}^{\infty} u_{z}^{m}=\sum_{m=0}^{\infty} A_{m}^{P+} U_{z}^{m}(z) e^{i k_{m}\left(x-c_{m} t\right)}, \\
& \tilde{u}_{x}=\sum_{m=0}^{\infty} \tilde{u}_{x}^{m}=\sum_{m=0}^{\infty} A_{m}^{P+} \tilde{U}_{x}^{m}(z) e^{i k_{m}\left(x-c_{m} t\right)}, \\
& \tilde{u}_{z}=\sum_{m=0}^{\infty} \tilde{u}_{z}^{m}=-i \sum_{m=0}^{\infty} A_{m}^{P+} \tilde{U}_{z}^{m}(z) e^{i k_{m}\left(x-c_{m} t\right)} .
\end{aligned}
$$


Reciprocity theorem in general provides a relation between displacements, tractions and body forces of two different loading states. Based on this relation of two states, solutions of scattered wave fields of the actual state can be derived. The original idea was discussed in [17], mostly for the cases of a solid half-space and a solid plate. The reciprocity approach was recently used for computation of guided wave motions in layered structures $[19,24]$. The verification of this approach was reported in $[18,28,29]$. For a three-material body, the reciprocity relation follows from Eq. (3) of [22]

$$
\begin{aligned}
& \int_{\hat{\Omega}}\left(\hat{f}_{j}^{A} \hat{u}_{j}^{B}-\hat{f}_{j}^{B} \hat{u}_{j}^{A}\right) d \hat{\Omega}+\int_{\Omega}\left(f_{j}^{A} u_{j}^{B}-f_{j}^{B} u_{j}^{A}\right) d \Omega+\int_{\tilde{\Omega}}\left(\tilde{f}_{j}^{A} \tilde{u}_{j}^{B}-\tilde{f}_{j}^{B} \tilde{u}_{j}^{A}\right) d \tilde{\Omega} \\
& =\int_{\hat{S}}\left(\hat{\tau}_{i j}^{B} \hat{u}_{j}^{A}-\hat{\tau}_{i j}^{A} \hat{u}_{j}^{B}\right) \hat{n}_{i} d \hat{S}+\int_{S}\left(\tau_{i j}^{B} u_{j}^{A}-\tau_{i j}^{A} u_{j}^{B}\right) n_{i} d S+\int_{\tilde{S}}\left(\tilde{\tau}_{i j}^{B} \tilde{u}_{j}^{A}-\tilde{\tau}_{i j}^{A} \tilde{u}_{j}^{B}\right) \tilde{n}_{i} d \tilde{S},
\end{aligned}
$$

where $\hat{f}, f$ and $\tilde{f}$ represent the forces applied on the upper fluid, the solid and the lower fluid, respectively, $\hat{S}, S$ and $\tilde{S}$ describes the contours around $\hat{\Omega}, \Omega$ and $\tilde{\Omega}$ without the interfaces with other bodies, respectively, while $\hat{n}_{i}, n_{i}$ and $\tilde{n}_{i}$ are normal vectors along $\hat{S}, S$ and $\tilde{S}$, respectively, see Fig. 3 . Also, superscripts $A$ and $B$ indicate two elastodynamic states. The actual state $A$ is the field generated by $f_{z}^{A}$ while the virtual state $B$ is the guided wave field propagating in the trilayered plate.

We should now choose an appropriate virtual state $B$ based on the expressions of free guided wave fields in Eqs. (17)-(36). State $B$ is set to include only a single wave mode with amplitude $B_{n}$. The negative $x$-direction state $B$ is written as

$$
\begin{aligned}
& \hat{u}_{x}^{n}=-B_{n} \hat{U}_{x}^{n}(z) e^{-i k_{n}\left(x+c_{n} t\right),} \\
& \hat{u}_{z}^{n}=-i B_{n} \hat{U}_{z}^{n}(z) e^{-i k_{n}\left(x+c_{n} t\right),} \\
& u_{x}^{n}=-B_{n} U_{x}^{n}(z) e^{-i k_{n}\left(x+c_{n} t\right),} \\
& u_{z}^{n}=B_{n} U_{z}^{n}(z) e^{-i k_{n}\left(x+c_{n} t\right),} \\
& \tilde{u}_{x}^{n}=-B_{n} \tilde{U}_{x}^{n}(z) e^{-i k_{n}\left(x+c_{n} t\right),} \\
& \tilde{u}_{z}^{n}=-i B_{n} \tilde{U}_{z}^{n}(z) e^{-i k_{n}\left(x+c_{n} t\right)} .
\end{aligned}
$$

The next step is to replace the expressions of states $A$ and $B$ into Eq. (44). The lefthand side of Eq. (44) can be simplified because the loading is applied only at $\left(x_{0}, z_{0}\right)$. Note that the right-hand side of Eq. (44) vanishes when state $A$ and state $B$ are in same direction. Therefore, there is only contribution from the counter-propagating waves, see $[17,19]$ for details. Since free boundary conditions are applied on the top and the bottom of the trilayered plate, there is no contribution of the integration. Moreover, using the orthogonality condition, derived in Appendix B, Eq. (B.10), the right-hand side of Eq. (44) cancels out for $m \neq n$. It should be noted that the time-harmonic load can be arbitrarily applied at any position in the structure. Without loss of generality, the load is applied in the solid layer $\Omega$. We finally find, after some manipulation, the amplitude of guided 
waves in the positive $x$-direction as

$$
A_{n}^{P+}=\frac{-i P U_{z}^{n}\left(z_{0}\right) e^{-i k_{n} x_{0}}}{2\left(\hat{\mu} \hat{I}_{n}+\lambda I_{n}+\tilde{\mu} \tilde{I}_{n}\right)}
$$

where

$$
\begin{aligned}
& \hat{I}_{n}=i k_{n} \int_{0}^{\hat{h}}\left[\hat{T}_{x x}^{n}(z) \hat{U}_{x}^{n}(z)\right] d z \\
& I_{n}=i k_{n} \int_{-h}^{0}\left[T_{x x}^{n}(z) U_{x}^{n}(z)+T_{x z}^{n}(z) U_{z}^{n}(z)\right] d z, \\
& \tilde{I}_{n}=i k_{n} \int_{-h+\tilde{h}}^{-h}\left[\tilde{T}_{x x}^{n}(z) \tilde{U}_{x}^{n}(z)\right] d z .
\end{aligned}
$$

Note that $\hat{I}_{n}, I_{n}$ and $\tilde{I}_{n}$ are connected to the guided wave of mode $n$. They are obtained from $\hat{I}_{m n}, I_{m n}$ and $\tilde{I}_{m n}$ expressed in Eqs. (B.7)-(B.9) of Appendix B, respectively, as $m=n$.

If a virtual wave of mode $n$ (state $B$ ) in the positive $x$-direction is chosen, we obtain

$$
A_{n}^{P-}=\frac{-i P U_{z}^{n}\left(z_{0}\right) e^{i k_{n} x_{0}}}{2\left(\hat{\mu} \hat{I}_{n}+\lambda I_{n}+\tilde{\mu} \tilde{I}_{n}\right)} .
$$

Similarly, for a horizontal load of the form

$$
f_{x}^{A}=Q \delta\left(z-z_{0}\right) \delta\left(x-x_{0}\right) e^{-i k c t},
$$

we find

$$
\begin{aligned}
& A_{n}^{Q+}=\frac{-Q U_{x}^{n}\left(z_{0}\right) e^{-i k_{n} x_{0}}}{2\left(\hat{\mu} \hat{I}_{n}+\lambda I_{n}+\tilde{\mu} \tilde{I}_{n}\right)}, \\
& A_{n}^{Q-}=\frac{Q U_{x}^{n}\left(z_{0}\right) e^{i k_{n} x_{0}}}{2\left(\hat{\mu} \hat{I}_{n}+\lambda I_{n}+\tilde{\mu} \tilde{I}_{n}\right)} .
\end{aligned}
$$

Eqs. (51) and (55) represent the amplitudes of guided waves of mode $n$ generated by the application of a vertical time-harmonic load of magnitude $P$ at $\left(x_{0}, z_{0}\right)$ obtained in closed-form solution. Similarly, Eqs. (57) and (58) express the amplitudes due to a horizontal force of magnitude $Q$ at $\left(x_{0}, z_{0}\right)$.

As an example, we calculate these expressions for a trilayered model which includes a $3 \mathrm{~mm}$-thick water layer, a $5 \mathrm{~mm}$-thick aluminum layer and a $10 \mathrm{~mm}$-thick water layer. The material properties are given in Tab. 1. In this model, water is used to mimic human soft-tissue and marrow while the aluminum is used to mimic human cortical bone (see Fig. 2 of Ref. [7]). For this calculation, the vertical load is applied at the interface of the upper fluid layer and the solid layer with a magnitude chosen as $P=\mu / 2$. Also, low frequencies ranging from 5 to $40 \mathrm{kHz}$ were used so only the lowest guided wave modes 
$A_{0}$ and $S_{0}$ will be generated. The normalized amplitudes of the lowest wave modes at the interface of the upper fluid and the solid are displayed in Fig. 4.

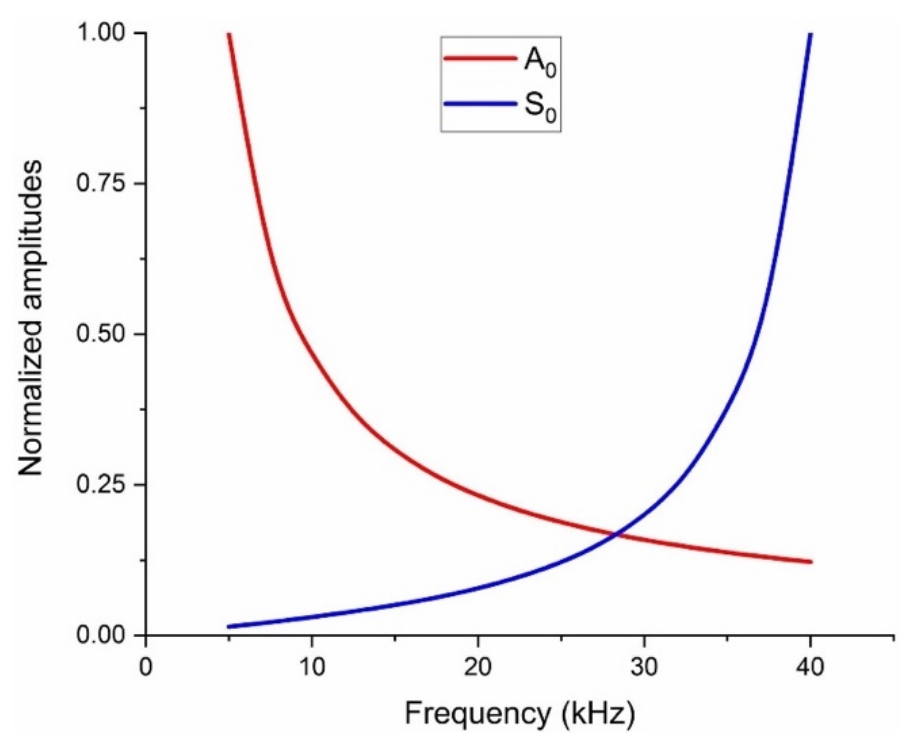

Fig. 4. Amplitudes of the lowest wave modes due to time-harmonic loading

\section{CONCLUSIONS}

A theoretical approach for guided wave motions in an isotropic solid plate coupled with two fluid layers has been proposed in this article. We have derived the characteristic equation and obtained velocity dispersion curves for a three-layered plate. In order to perform reciprocity application, the expressions of free guided waves have been introduced. It has also analytically computed the amplitudes of guided wave modes subjected to a time-harmonic load applied in the solid layer. As an example, we have presented the results for normalized amplitudes of the lowest wave modes. The theoretical predictions obtained in the current work will be beneficial in building models for a cortical bone-mimicking plate coupled with soft-tissue layers and, in general, useful for further applications in bone quantitative ultrasound.

\section{ACKNOWLEDGMENT}

We would like to acknowledge the International Center of Physics for support of this research (Grant No. ICP.2020.12).

\section{REFERENCES}

[1] L. H. Le, Y. J. Gu, Y. Li, and C. Zhang. Probing long bones with ultrasonic body waves. Applied Physics Letters, 96, (11), (2010). https://doi.org/10.1063/1.3300474. 
[2] V.-H. Nguyen and S. Naili. Ultrasonic wave propagation in viscoelastic cortical bone plate coupled with fluids: a spectral finite element study. Computer Methods in Biomechanics and Biomedical Engineering, 16, (9), (2013), pp. 963-974. https://doi.org/10.1080/10255842.2011.645811.

[3] P. Laugier and G. Haïat. Bone quantitative ultrasound. Springer, (2010).

[4] P. Laugier. Quantitative ultrasound of bone: looking ahead. Joint Bone Spine, 73, (2), (2005), pp. 125-128. https://doi.org/10.1016/j.jbspin.2005.10.012.

[5] G. Lowet and G. Van der Perre. Ultrasound velocity measurement in long bones: measurement method and simulation of ultrasound wave propagation. Journal of Biomechanics, 29, (10), (1996), pp. 1255-1262. https://doi.org/10.1016/0021-9290(96)00054-1.

[6] P. H. F. Nicholson, P. Moilanen, T. Kärkkäinen, J. Timonen, and S. Cheng. Guided ultrasonic waves in long bones: modelling, experiment and in vivo application. Physiological Measurement, 23, (4), (2002). https://doi.org/10.1088/0967-3334/23/4/313.

[7] V.-H. Nguyen, T. N. H. T. Tran, M. D. Sacchi, S. Naili, and L. H. Le. Computing dispersion curves of elastic/viscoelastic transversely-isotropic bone plates coupled with soft tissue and marrow using semi-analytical finite element (SAFE) method. Computers in Biology and Medicine, 87, (2017), pp. 371-381. https://doi.org/10.1016/j.compbiomed.2017.06.001.

[8] J. Achenbach. Wave propagation in elastic solids. North-Holland Publishing Company, (1973).

[9] J. L. Rose. Ultrasonic guided waves in solid media. Cambridge University Press, (2014).

[10] S. Banerjee and C. B. Pol. Theoretical modeling of guided wave propagation in a sandwich plate subjected to transient surface excitations. International Journal of Solids and Structures, 49, (23-24), (2012), pp. 3233-3241. https://doi.org/10.1016/j.ijsolstr.2012.06.022.

[11] A. Chattopadhyay, P. Singh, P. Kumar, and A. K. Singh. Study of Love-type wave propagation in an isotropic tri layers elastic medium overlying a semi-infinite elastic medium structure. Waves in Random and Complex Media, 28, (4), (2018), pp. 643-669. https://doi.org/10.1080/17455030.2017.1381778.

[12] C. L. Yapura and V. K. Kinra. Guided waves in a fluid-solid bilayer. Wave Motion, 21, (1), (1995), pp. 35-46. https://doi.org/10.1016/0165-2125(94)00043-5.

[13] E. Kausel, P. Malischewsky, and J. Barbosa. Osculations of spectral lines in a layered medium. Wave Motion, 56, (2015), pp. 22-42. https://doi.org/10.1016/j.wavemoti.2015.01.004.

[14] E. Kausel. Fundamental solutions in elastodynamics: a compendium. Cambridge University Press, (2006).

[15] R. K. N. D. Rajapakse and Y. Wang. Green's functions for transversely isotropic elastic half space. Journal of Engineering Mechanics, 119, (9), (1993), pp. 1724-1746. https://doi.org/10.1061/(asce)0733-9399(1993)119:9(1724).

[16] W. M. Ewing, W. S. Zardetzky, and F. Press. Elastic waves in layered media. McGraw-Hill, (1957).

[17] J. A. Achenbach and J. D. Achenbach. Reciprocity in elastodynamics. Cambridge University Press, (2003).

[18] H. Phan, Y. Cho, and J. D. Achenbach. Validity of the reciprocity approach for determination of surface wave motion. Ultrasonics, 53, (3), (2013), pp. 665-671. https://doi.org/10.1016/j.ultras.2012.09.007.

[19] H. Phan, T. Q. Bui, H. T.-L. Nguyen, and C. V. Pham. Computation of interface wave motions by reciprocity considerations. Wave Motion, 79, (2018), pp. 10-22. https://doi.org/10.1016/j.wavemoti.2018.02.008. 
[20] H. Phan, Y. Cho, and J. D. Achenbach. Application of the reciprocity theorem to scattering of surface waves by a cavity. International Journal of Solids and Structures, 50, (24), (2013), pp. 4080-4088. https://doi.org/10.1016/j.ijsolstr.2013.08.020.

[21] P. T. Nguyen, H. Nguyen, D. Le, and H. Phan. A model for ultrasonic guided waves in a cortical bone plate coupled with a soft-tissue layer. In AIP Conference Proceedings, Vol. 2102, (2019). https://doi.org/10.1063/1.5099773.

[22] H. Phan, Y. Cho, C. V. Pham, H. Nguyen, and T. Q. Bui. A theoretical approach for guided waves in layered structures. In AIP Conference Proceedings, Vol. 2102, (2019). https://doi.org/10.1063/1.5099777.

[23] P.-T. Nguyen and H. Phan. A theoretical study on propagation of guided waves in a fluid layer overlying a solid half-space. Vietnam Journal of Mechanics, 41, (1), (2019), pp. 51-62. https://doi.org/10.15625/0866-7136/12710.

[24] H. Phan, Y. Cho, Q. H. Le, C. V. Pham, H. T.-L. Nguyen, P. T. Nguyen, and T. Q. Bui. A closed-form solution to propagation of guided waves in a layered half-space under a timeharmonic load: An application of elastodynamic reciprocity. Ultrasonics, 96, (2019), pp. 40-47. https://doi.org/10.1016/j.ultras.2019.03.015.

[25] H. Phan, Y. Cho, and W. Li. A theoretical approach to multiple scattering of surface waves by shallow cavities in a half-space. Ultrasonics, 88, (2018), pp. 16-25. https://doi.org/10.1016/j.ultras.2018.02.018.

[26] J. Lee, V. Ngo, H. Phan, T. Nguyen, D. K. Dao, and Y. Cho. Scattering of surface waves by a three-dimensional cavity of arbitrary shape: analytical and experimental studies. Applied Sciences, 9, (24), (2019). https://doi.org/10.3390/app9245459.

[27] W. Liu, Y. Cho, H. Phan, and J. D. Achenbach. Study on the scattering of 2-D Rayleigh waves by a cavity based on BEM simulation. Journal of Mechanical Science and Technology, 25, (3), (2011), pp. 797-802. https://doi.org/10.1007/s12206-011-0133-5.

[28] D. K. Dao, V. Ngo, H. Phan, C. V. Pham, J. Lee, and T. Q. Bui. Rayleigh wave motions in an orthotropic half-space under time-harmonic loadings: A theoretical study. Applied Mathematical Modelling, 87, (2020), pp. 171-179. https://doi.org/10.1016/j.apm.2020.06.006.

[29] H. Phan, Y. Cho, and J. D. Achenbach. Verification of surface wave solutions obtained by the reciprocity theorem. Ultrasonics, 54, (7), (2014), pp. 1891-1894. https://doi.org/10.1016/j.ultras.2014.05.003. 


\section{APPENDIX A. EXPRESSIONS OF GUIDED WAVES IN THE TRILAYERED STRUCTURE}

Eight-by-eight matrix

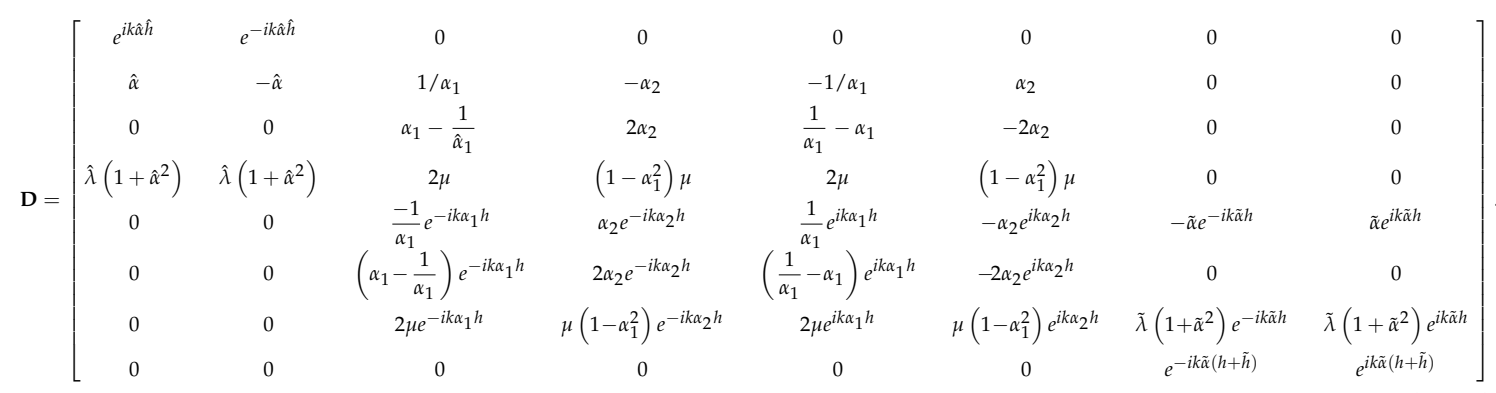

Dimensionless quantities

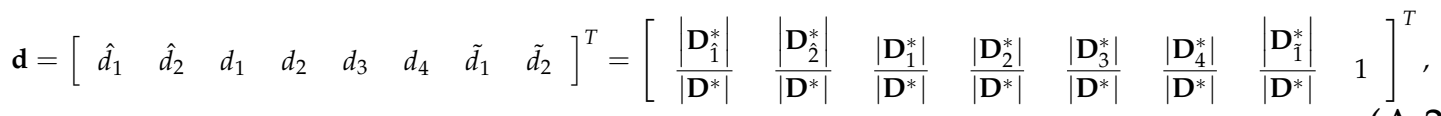

where

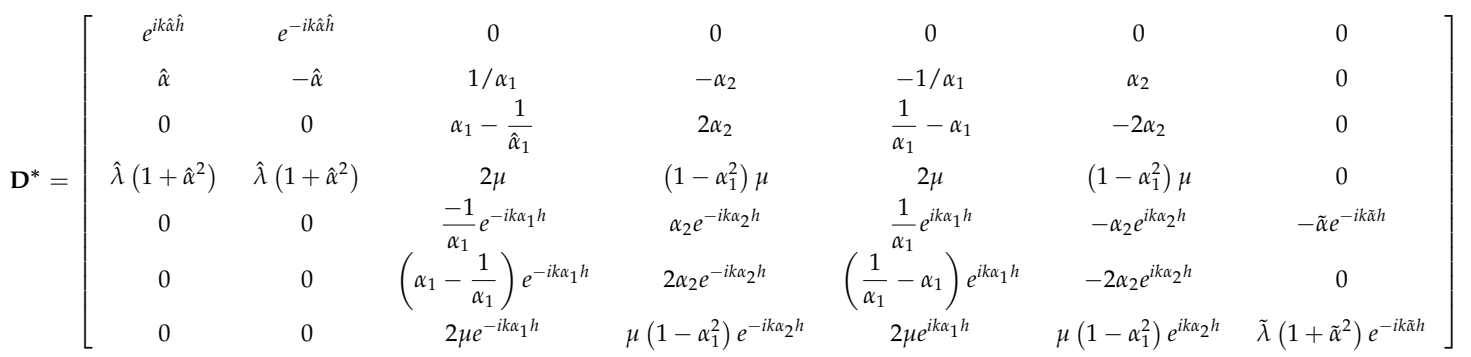

$$
\begin{aligned}
& \text { and } \mathbf{k}=\left[\begin{array}{c}
0 \\
0 \\
0 \\
0 \\
-\tilde{\alpha} e^{i k \tilde{\alpha} h} \\
0 \\
-\tilde{\lambda}\left(1+\tilde{\alpha}^{2}\right) e^{i k \tilde{\alpha} h}
\end{array}\right]
\end{aligned}
$$

with $\mathbf{D}_{i}^{*}$ formed by replacing the column $i$ of matrix $\mathbf{D}^{*}$ by $\mathbf{k}$.

\section{APPENDIX B. ORTHOGONALITY CONDITION}

We derive here an orthogonality condition for counter-propagating guided waves in the trilayered plate using the reciprocity relation given in Eq. (44). The condition is related to two free guided waves of mode $m$ with wavenumber $k_{m}$ (state $A$ ) and mode $n$ with wavenumber $k_{n}$ (state $B$ ). The domain is defined by $a \leq x \leq b,-(h+\tilde{h}) \leq z \leq \hat{h}$ as demonstrated in Fig. 3. With the absence of a force term, the left-hand side of Eq. (44) 
vanishes. This leads to

$$
\int_{\hat{S}}\left(\hat{\tau}_{i j}^{B} \hat{u}_{j}^{A}-\hat{\tau}_{i j}^{A} \hat{u}_{j}^{B}\right) \hat{n}_{i} d \hat{S}+\int_{S}\left(\tau_{i j}^{B} u_{j}^{A}-\tau_{i j}^{A} u_{j}^{B}\right) n_{i} d S+\int_{\tilde{S}}\left(\tilde{\tau}_{i j}^{B} \tilde{u}_{j}^{A}-\tilde{\tau}_{i j}^{A} \tilde{u}_{j}^{B}\right) \tilde{n}_{i} d \tilde{S}=0 .
$$

Applying free boundary conditions on the top and the bottom of the trilayered plate, Eq. (B.1) becomes

$$
\left.\int_{0}^{\hat{h}} \hat{F}_{A B}^{m n}\right|_{x=a} d z+\left.\int_{-h}^{0} F_{A B}^{m n}\right|_{x=a} d z+\left.\int_{-(h+\tilde{h})}^{-h} \tilde{F}_{A B}^{m n}\right|_{x=a} d z=\left.\int_{0}^{\hat{h}} \hat{F}_{A B}^{m n}\right|_{x=b} d z+\left.\int_{-h}^{0} F_{A B}^{m n}\right|_{x=b} d z+\left.\int_{-(h+\tilde{h})}^{-h} \tilde{F}_{A B}^{m n}\right|_{x=b} d z,
$$

where $\hat{F}_{A B}^{m n}, F_{A B}^{m n}$ and $\tilde{F}_{A B}^{m n}$ are expressed as

$$
\begin{aligned}
& \hat{F}_{A B}^{m n}=\hat{\tau}_{x x}^{B n} \hat{u}_{x}^{A m}-\hat{\tau}_{x x}^{A m} \hat{u}_{x}^{B n}, \\
& F_{A B}^{m n}=\tau_{x x}^{B n} u_{x}^{A m}+\tau_{x z}^{B n} u_{z}^{A m}-\tau_{x x}^{A m} u_{x}^{B n}-\tau_{x z}^{A m} u_{z}^{B n}, \\
& \tilde{F}_{A B}^{m n}=\tilde{\tau}_{x x}^{B n} u_{x}^{A m}-\tilde{\tau}_{x x}^{A m} \tilde{u}_{x}^{B n} .
\end{aligned}
$$

Using the displacement and stress expressions of the states $A$ and $B$ into Eq. (B.2), after some manipulation, yields

$$
\left(e^{i\left(k_{m}-k_{n}\right) a}-e^{i\left(k_{m}-k_{n}\right) b}\right)\left(\hat{\mu} \hat{I}_{m n}+\mu I_{m n}+\tilde{\mu} \tilde{I}_{m n}\right)=0,
$$

where

$$
\begin{aligned}
& \hat{I}_{m n}=\frac{1}{2} \int_{0}^{\hat{h}} i\left[k_{n} \hat{T}_{x x}^{n}(z) \hat{U}_{x}^{m}\left(z+k_{m} \hat{T}_{x x}^{m}(z) \hat{U}_{x}^{n}(z)\right] d z,\right. \\
& I_{m n}=\frac{1}{2} \int_{-h}^{0} i\left[k_{n}\left(T_{x x}^{n}(z) U_{x}^{m}(z)-T_{x z}^{n}(z) U_{z}^{m}(z)\right)+k_{m}\left(T_{x x}^{m}(z) U_{x}^{n}(z)-T_{x z}^{m}(z) U_{z}^{n}(z)\right)\right] d z, \\
& \tilde{I}_{m n}=\frac{1}{2} \int_{-(h+\tilde{h})}^{-h} i\left[k_{n} \tilde{T}_{x x}^{n}(z) \tilde{U}_{x}^{m}(z)+k_{m} \tilde{T}_{x x}^{m}(z) \tilde{U}_{x}^{n}(z)\right] d z .
\end{aligned}
$$

Note that Eq. (B.6) must be satisfied for arbitrary values of $a$ and $b$. Clearly, it is satisfied if $m=n$. When $m \neq n$, it can be satisfied only if $\hat{\mu} \hat{I}_{m n}+\mu I_{m n}+\tilde{\mu} \tilde{I}_{m n}=0$. For $m \neq n$, thus, we obtain the orthogonality condition of guided waves in a trilayered plate as

$$
\hat{\mu} \hat{I}_{m n}+\mu I_{m n}+\tilde{\mu} \tilde{I}_{m n}=0 .
$$

It should be noted that as $m=n$, quantities $\hat{I}_{n}, I_{n}$ and $\tilde{I}_{n}$ expressed in Eqs. (52)-(54) are $\hat{I}_{m n}, I_{m n}$ and $\tilde{I}_{m n}$ given in Eqs. (B.7)-(B.9). 